\title{
Indépendance, Savoir Aborigène et Environnement en Nouvelle-Calédonie
}

\author{
Donna Winslow
}

\section{Introduction}

Max Weber est l'auteur qui a mis en contraste les rationalités formelle et substantive. Selon lui, la rationalité formelle fait appel à la notion de moyens, c'est-à-dire de moyens de plus en plus efficaces pour accomplir les choses. La rationalité formelle peut être différenciée d'un choix de buts rationnels que Weber appelle rationalité substantive et qui peut varier selon les valeurs impliquées. "La rationalité formelle implique essentiellement la calculabilité des moyens et des procédures alors que la rationalité substantive s'appuie sur la valeur (d'un point de vue explicitement défini) des fins ou résultats."1

En Nouvelle-Calédonie, un Territoire français d'Outre-mer du Pacifique Sud, la connaissance indigène était ancrée dans une approche substantive de l'environnement naturel, caractérisé par une conviction dans les valeurs intrinsèques d'un objet ou d'un acte. Quand le territoire fut annexé par la France en 1853, les relations capitalistes de productions ont été introduites avec le développement colonial basé sur l'immigration, l'exploitation des ressources minières et l'élevage. Ceci a, à son tour, introduit la rationalité formelle, caractérisée par le calcul des moyens vers une fin. L'environnement naturel est devenu alors, un moyen ou un instrument de poursuite consciencieuse du profit pour les colonisateurs. Au même moment, la majorité indigène kanake ${ }^{2}$ vivait dans des réserves, continuait les activités vivrières et appréciait toujours les valeurs intrinsèques de la nature. Ils ont participé à l'économie coloniale comme travailleurs forcés, où chaque village avait pour obligation d'envoyer des hommes pour les travaux publics, ou plus récemment comme ouvriers salariés.

Toutefois, dans les vingt dernières années une nouvelle élite de leaders kanaks a émergé, elle promouvait la participation au développement économique comme la production du nickel. J'ai commencé ce document avec l'intention d'argumenter sur le fait

1. Roger Brubaker, The Limits of Rationality. London: George Allen \& Unwin, 1984, p. 36, traduction.

2. Le terme "Canaque" fut introduit en Nouvelle-Calédonie par des équipages polynésiens pendant la période de premier contact avec des sociétés européennes. Dans ce contexte le terme était souvent utilisé de façon péjorative pour désigner les mélanésiens de la Nouvelle-Calédonie. Ce n'est que dans les années 70 que les autochtones ont changé l'appellation pour "Kanak" (voir Chesneaux, J. Transpacifiques. Observations et considérations diverses sur les terres et archipels du Grand Océan. Paris: La Découverte, 1988, pour plus de détails). Kanak est maintenant un terme valorisant, associé aux revendications nationalistes des autochtones de l'archipel. Afin de simplifier ce texte nous avons utilisé le mot "Kanak" pour désigner les autochtones de la Nouvelle-Calédonie pendant la période qui précède le développement du mouvement indépendantiste. 
que les dirigeants kanaks ont développé une nouvelle vision rationnelle de leur environnement, en développant leurs richesses minières d'une façon rationnelle. Cependant, après avoir achevé la recherche, ${ }^{3} \mathrm{j}$ 'en suis venue à conclure que le point de vue des Kanaks sur le développement de leur environnement demeure substantif au lieu d'être devenu formel. La rationalité substantive fait primer la valeur du but ou du résultat sur la calculabilité des moyens et des procédures. Pour le Front d'Indépendance Kanak, l'utilisation de l'environnement naturel est désormais perçue comme une manière d'aboutir à un but spécifique - La souveraineté. En Nouvelle-Calédonie, les dirigeants kanaks développent et contrôlent les ressources de leur pays, par exemple les mines de nickel, avec les buts précis de construire les bases de l'indépendance et le développement de la collectivité plutôt que de délibérément poursuivre des intérêts personnels dans les opportunités du marché.

La structure de ce document est de comparer et d'établir un contraste entre la société des Kanaks et la société des colons français. Cela reflète néanmoins la polarisation qui existe dans les territoires colonisés. Dans le climat politique actuel deux communautés réclament leur légitimité et se trouvent face à face. La légitimité des uns se fonde sur le droit de la conquête et de l'occupation réclamée par les Européens et leurs travailleurs asiatiques et polynésiens s'appuyant sur leur domination politique et économique des îles. Cette population est solidement rattachée à la France et se concentre dans la région urbaine de Nouméa, la capitale du territoire. La légitimité des autres, réclamée par les kanaks comme étant les premiers occupants des terres, est fondée sur leur rythme de vie qui reste, pour la plupart, rural et orienté sur la communauté. "Ce qui se passe en Nouvelle-Calédonie aujourd'hui" d' après Léopold Jorédie, Président kanak de la Province Nord, " $n$ 'est rien de plus que la confrontation entre deux idées différentes." 4 De telles oppositions ne sont pas isolées, elles existent dans de plus larges systèmes. En NouvelleCalédonie le système est colonial et divise Kanaks et Non-Kanaks par la distribution inéquitable de la terre, des ressources et du pouvoir.

\section{La société traditionnelle kanake}

La Nouvelle-Calédonie est située dans 1'Océan Pacifique, à 1500 kilomètres à l'est de l'Australie et à 1700 kilomètres au nord de la Nouvelle-Zélande. Elle est à 22,000 kilomètres de la France. Sa superficie totale est de 19,103 kilomètres carrés. Il y a une grande île, la Grande Terre, avec une superficie de 16,750 kilomètres carrés, et plusieurs petites îles qui totalisent 2353 kilomètres carrés. Ces petites îles sont les Iles Loyautés (Ouvéa, Maré, Lifou et Tiga), l'archipel de Bélep, l'Ile des Pins et l'Ile Huon.

3. J'ai commencé les recherches sur le terrain en Nouvelle-Calédonie en 1984 alors que j'étais étudiante au doctorat. Depuis, j’y suis retournée régulièrement. Mon expérience làbas s'étend sur plus de dix ans. Les recherches sur le terrain ayant trait à cet article ont été menées de juin à octobre 1993.

4. Léopold Jorédie, communication personnelle, mai 1989. 


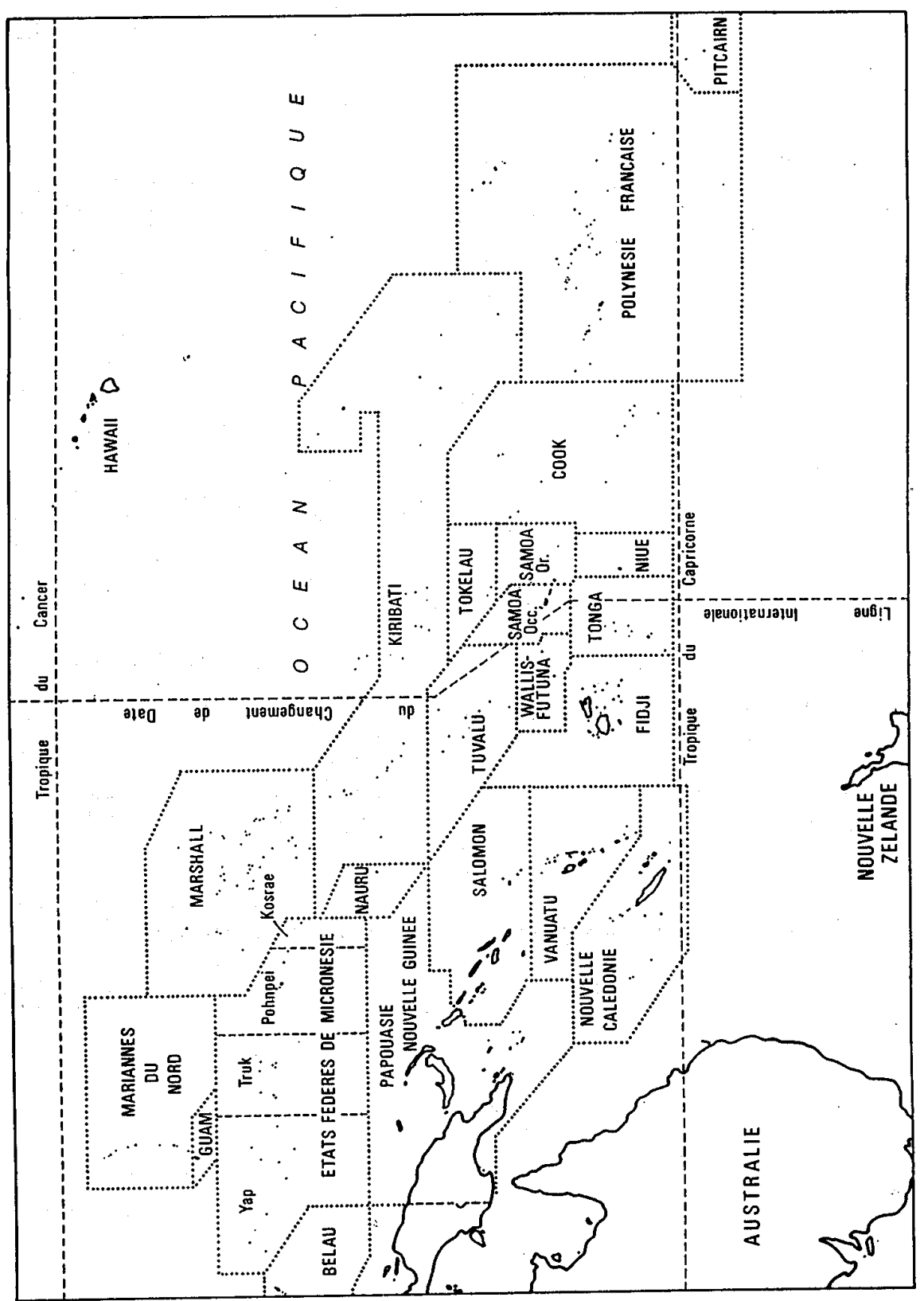

Figure 1. Carte de la Région de la Nouvelle-Calédonie.

Source: East West Center, Pacific Islands Development Program and the Hawai'i Geographic Society, Honolulu, Hawai'i, 1992.

D'après les sources archéologiques, les premiers ancêtres des Kanaks sont venus en Nouvelle-Calédonie du Sud-Est Asiatique il y a 5 ou 6000 ans. Il y avait aussi des vagues de colonisations venues d'autres parties de la Mélanésie, particulièrement des Iles Salomon et Vanuatu. Ces premiers colons ont apporté avec eux l'agriculture sur brûlis et la 
jachère, les techniques d'irrigations, les outils complexes en pierres polies, la poterie et la construction des bateaux de pêche à doubles ponts, Les plantes médicinales, les réserves de nourritures, l'igname, le taro, le fruit de l'arbre à pain, la noix de coco et les arbres dont l'écorce est utilisée comme matériel de tissage.

Lorsque le Capitaine Cook visita la Nouvelle-Calédonie en 1774, il découvrit que les îles étaient peuplées par un peuple mélanésien (les Kanaks) dispersés le long des rivières dans les vallées et les villages côtiers. Les communautés étaient composées de huttes au toit de chaume rondes pour les hommes et les femmes, des cuisines collectives rectangulaires, de maisons de rencontre oblongues et d'ateliers de formes diverses. Chaque femme avait sa propre hutte où elle élevait ses enfants. Ces structures étaient construites en bordure d'une grande case utilisée par le chef et les hommes adultes. Cette grande case était érigée sur un tertre avec une allée centrale bordée de palmiers de cocotiers et de pins tropicaux et flanquée de deux allées plus petites. L'allée centrale était utilisée comme lieu de cérémonie collective pour des activités telles que les discours publics et la redistribution de l'igname; alors que les allées secondaires étaient utilisées pour les rituels plus intimes comme les cérémonies d'échange de monnaie-coquillage. Traditionnellement, cet espace social de résidences familiales, de terres agricoles, de canaux d'irrigations, de territoires de chasse et de réunion, constituait la base pour le rituel, l'économie, la politique et l'action sociale.

Les familles nucléaires et étendues formaient l'unité de production de base avec les voisins et alliés appelés à l'aide en fonction de la taille de la tâche. Traditionnellement, chaque communauté locale était intégrée dans un système politique et géographique plus grande par l'intermédiaire d'alliance et d'échange. Les cérémonies d'échanges renforçaient l'identité politique et sociale des familles, les unes envers les autres. La division du travail se faisait en fonction du genre et de l'âge. Le travail était organisé selon un calendrier rituel saisonnier contrôlé par les aînés. Les repas étaient pris en groupe et le travail fait en groupes.

Les ignames étaient considérées comme "nobles" et on s'en servait dans les cérémonies d'échanges. Le cycle annuel d'igname définissait le rythme de l'année kanake. La pêche était une activité régulière pour les installations en bordure de mer ou de rivières. Dans la forêt les kanaks cueillaient des fruits, des noix et des bourgeons de palmiers. Hommes et femmes chassaient les fruits de mer, seuls ou collectivement utilisant des lances, des cannes à pêche et des filets. Les hommes chassaient le peu qu'il y avait oiseaux, chauve-souris et rats - avec des lances. Ils construisaient des huttes et des bateaux, et veillaient à la production d'ignames, aux travaux d'irrigation et aux tâches d'agricultures les plus dures. ${ }^{5}$ Les femmes kanakes ramassaient le bois et l'eau, veillaient sur les enfants et faisaient les tâches répétitives d'agriculture telle l'ensemensement. Les hommes travaillaient avec la pierre et le bois, à la confection d'outils et d'armes, et les femmes travaillaient avec l'argile et les fibres végétales à la fabrication de pots, de nattes, de paniers et de jupes en fibres.

La famille nucléaire faisait partie de la famille étendue (habituellement de trois générations). La lignée et le clan ne représentait pas les groupes territoriaux mais plutôt des unités patriarcales de plus en plus larges, partageant les mêmes rites et symboles, et les

5. C'est le Capitaine Cook qui a introduit le porc et le chien dans les îles et les autres européens ont introduit une variété d'espèces de plantes et d'animaux incluant le cerf et la vache. 
mêmes coutumes de mariage basées sur l'exogamie, la patrilocalité et le mariage entre cousins germains. Les aînés, têtes de lignées, étaient perçus comme les gardiens des relations sociales et symboliques qui unissaient les familles en alliances communales et régionales. La mémoire généalogique s'exprimait en séries de patronymes et de typonymes. Le système de patronymes fonctionnait pour régulariser le comportement entre les individus vivants. Chaque personne traçait sa lignée au dernier ascendant paternel connu (habituellement à travers trois générations) et collatéralement, aux descendants des frères du grand-père et de ses descendants. En plus de cela, la généalogie s'exprimait dans l'espace. Le système de noms propres était adjoint à un système de noms géographiques. Les familles étendues étaient rassemblées dans des groupes d'affiliation (lignées et clans) plus grands par référence à un lieu d'origine commun (tertre cérémonial). Ces sites se réfèrent aux tertres érigés dans les anciens camps avec la large habitation centrale utilisée par le chef et les hommes adultes.

Le point de référence du clan était le tertre fondé par le clan ancêtre, et chaque clan connaissait son histoire au travers des multiples générations marquées par la succession des lieux d'occupation des sites/tertres. L'histoire de chaque clan était une description de longues séries de déplacements et, à l'intérieur de chaque clan, les lignées étaient placées hiérarchiquement selon l'ancienneté de leur première résidence dans l'itinéraire généalogique du clan. La terre était ainsi la représentation matérielle du clan. ${ }^{6}$ Les récits du clan, qui sont encore énumérés dans les festivals, sont les chants de la marche commençant au tertre d'origine décrivant les voyages de chaques branches du clan. ${ }^{7}$

Dans la société kanake, le temps, tout comme la généalogie, avait une signification géographique. Dans les sociétés occidentales le temps est linéaire, séquencé, unidirectionnel, et des séries d'instants, de moments successifs. Le temps est une commodité dont l'un est “à court" et l'autre "à perdre." Pour les Kanaks, le temps occupe l'espace continuellement, selon le rythme des saisons. Au lieu d'utiliser le temps, les Kanaks habitent le temps. Le temps kanak est récurrent et fait la promotion du consensus, de la discussion et de la continuité. C'est à cause de la continuité de leur présence dans les îles que les Kanaks demandent la reconnaissance et les droits comme premiers occupants,

6. La terre avait aussi les qualités de la vie et de la mort. Les mythes du clan d'origine décrivent des ancêtres qui sont nés de roches, d'arbres, des sources ou des gouttes de pluies sur une feuille. Mais les Kanaks parlent aussi de la terre comme étant la chair des morts. Dans les anciennes coutumes d'inhumation les morts étaient exposés à l'air libre, placés dans les arbres ou les grottes et les ancêtres du clan retournaient ainsi à la terre. Ceci unit symboliquement le clan à la terre et à perpétuité pour que, si un clan quitte un territoire, il maintienne un lien spirituel au site d'origine. Un chef était responsable des relations avec les ancêtres de la terre et du maintien de bonnes relations entre le clan et les forces de l'univers. Il était un genre de calendrier vivant; le régulateur du cycle agraire. La plantation cérémonielle était faite dans son champ pour marquer le début de chaque saison et il était un point tournant dans un réseau complexe d'échange et essentiel à la vie rituelle de la société.

7. Ceci établit la légitimité de chaque lignée dans l'ordre social où leur nom est le premier site occupé par l'ancêtre de la lignée. Le statut social et la terre sont alloués selon le nom depuis l'accessibilité à la terre dans tous les sites occupés par les ancêtres. Les noms n'ont pas toujours été héréditaires et un enfant a pu recevoir le nom d'une lignée éteinte ou d'une lignée n'ayant pas d'héritiers mâles, pour que le nom puisse continuer. 
alors que les Français ont occupé la terre pour une période comparativement plus courte, comme colons récemment installés.

\section{Développement économique et environnement}

La base de l'économie de la Nouvelle-Calédonie est l'industrie du nickel, qui représente d'environ 80 pour-cent des exportations du territoire. La Nouvelle-Calédonie constitue une source importante de nickel. Elle contient 28 pour-cent des dépôts de nickel oxydé au monde et est le troisième pays en importance pour la production mondiale du nickel. Jusqu'à ce jour les Kanaks n'ont eu aucun contrôle sur les impacts miniers. La poursuite du profit par les Européens entraîne dans l'accumulation de déchets miniers et la dégradation de l'environnement. Ceci affecté grandement la population kanake vivant dans les vallées, en bas des sites miniers. Selon un militant kanak: "Les mineurs européens sont comme des voleurs - des voleurs qui viennent et volent dans votre jardin. Ils ne font pas attention au jardin. Ils remplissent leurs sacs et s'en vont."

Les mines de nickel à ciel ouvert ont commencé dans le territoire à la fin du siècle dernier. La nature de l'extraction du nickel représente une menace sérieuse pour l'environnement physique puisqu'elle détruit la végétation naturelle et enlève la couche supérieure du sol causant des érosions sévères sur les pentes escarpées des régions minières. Le minerai de nickel, se trouve sur les rochers "ultramafic" en zones de concentration sous une couche de matériaux exposés aux intempéries qui peut atteindre jusqu'à trente mètres d'épaisseur. Les extractions à grande échelle sur le faîte des montagnes et sur les plateaux sont réalisées en enlevant cette couche de surface. L'extraction s'effectue par coupes horizontales, formant d'énormes bancs de cinq à huit mètres de diamètre.

En 1943 et 1954-1955, l'exploitation était confinée aux crêtes des montagnes où le maximum de concentration de minerai était facilement accessible. Subséquemment, l'ensemble des portions centrales des masses montagneuses était coupé de 400 mètres audessus du niveau de la mer. Le minerai de nickel est extrait de plus en plus en profondeur à cause du tarissement des veines principales. Ceci occasionne de plus grands volumes d'agrégats dont on doit disposer. En moins de cent ans, 110 millions de tonnes de minerai ont été extraites, entraînant l'accumulation d'une masse de déchets au moins cinq fois plus importante en poids, et d'une volume atteignant de 220 à 280 millions de mètres cubes selon des estimations très conservatrices. ${ }^{9}$

Ces déchets miniers ont eu de sérieux impacts sur l'environnement naturel de la Nouvelle-Calédonie. Les dépôts de nickel se situent dans les portions supérieures des masses montagneuses. Ces montagnes sont extrêmement accidentées ayant des côtés très escarpés au pied de ces dernières, avec très peu de sites naturels pour disposer IN SITU des résidus. Les sentiers et les routes sont difficiles à établir et le matériel, sans attaches ne

8. Militant kanak, communication personnelle, 14 juin 1993.

9. M. Benezit, Report on Mining Pollution in New Caledonia, South Pacific Regional Environment Programme, Topic Review 01, Nouméa, Nouvelle-Calédonie: South Pacific Commission, 1981, pp. 1; J.F. Dupon, The Effects of Mining on the Environment of High Islands: a case study of nickel mining in New Caledonia, Nouméa: South Pacific Commission, 1986, pp.2,4. 
peut rester en place. Les travaux routiers, comme les sentiers aménagés à la hâte pour parvenir aux mines ou aux sites de prospection, ont aussi entraîné une érosion intense.

Le grattage et l'enlèvement de la surface du sol, nécessaire pour atteindre le minerai, détruit directement la végétation. La terre ainsi dégagée est d'autant plus vulnérable à l'érosion par l'eau. Cet effet est également intensifié par de fortes pluies saisonnières et des cyclones. Et lors de périodes de temps très sec, d'épais nuages de poussière sont produits par les opérations minières, particulièrement par le camionnage. Dans les mines, cette poussière est nuisible à la santé et à la sécurité des travailleurs. Dans les plaines, elle affecte les zones résidentielles et les fermes. Les pâturages bordant les routes deviennent moins fertiles sur une distance de 100 à 200 mètres de chaque côté de la route et le bétail évite ces régions. ${ }^{10}$

D'énormes quantités de cette terre fragile lavées par les pluies sont transportées dans les vallées. Rapetissant alors le lit des cours d'eau, elles provoquent des inondations sur les terres bordant les principales rivières et recouvre ainsi les terres agricoles fertiles de la vallée. Dans les vallées de ces rivières, les dépôts forment une épaisse couche qui enterre la végétation des berges et, très souvent, extermine cette végétation ainsi que la faune qui habite ces eaux.

Dans les années 80, les géographes Bird, Dubois et Iltis ont remarqué qu'en comparaison avec d'autres centres miniers ailleurs dans le monde, les impacts des mines à ciel ouvert aux sommets des montagnes de la Nouvelle-Calédonie ont été exceptionnellement sévères et étendus. ${ }^{11}$ Selon Dupon, la Nouvelle-Calédonie offre un exemple spectaculaire des dommages environnementaux résultant d'une exploitation minière intense et incontrôlée. ${ }^{12}$ Certainement, le territoire offre un exemple frappant des dommages pouvant être infligés à l'environnement d'une île du Pacifique par des activités minières peu contrôlées.

\section{Le rationalisme des sociétés occidentales selon Weber comparé à la société traditionnelle kanake}

Selon Weber, les civilisations occidentales sont différentes de toutes les autres dans leurs dimensions spécifiques et dans leur rationalisme particulier. ${ }^{13}$ Ici, nous examinerons le rationalisme occidental selon trois critères : la rationalité de l'économie capitaliste, de l'administration bureaucratique, et de l'orientation interne et nous les comparerons aux

10. M. Benezit, Report on Mining Pollution in New Caledonia, South Pacific Regional Environment Programme, Topic Review 01, Nouméa, Nouvelle-Calédonie: South Pacific Commission, 1981, pp. 1,4,6.

11. E. Bird; J.-P. Dubois; et J. Iltis, The Impacts of Opencast Mining on the Rivers and Coasts of New Caledonia, Tokyo: United Nations University Press, 1984, pp. 49, traduction.

12. J.F. Dupon, The Effects of Mining on the Environment of High Islands: a case study of nickel mining in New Caledonia, Nouméa: South Pacific Commission, 1986, pp.1, traduction.

13. M. Weber, "Author's Introduction." Dans: The Protestant Ethic and the Spirit of Caplitalism, Traduction de Talcott Parsons. New York: Scribner's, 1958, p.26, traduction. 
traditions kanakes pour mieux comprendre comment les visions traditionnelles kanakes continuent à persister malgré la participation des Kanaks dans les structures occidentales.

\title{
a) la rationalité de l'économie capitaliste
}

Commençons par l'examen du premier critère - la rationalité de l'économie capitaliste. Avec le capitalisme, les transactions de marché sont déterminées entièrement par la "poursuite intentionnelle d'intérêts," et par "une orientation de commodité."14 Selon Bruebaker, un spécialiste de Weber, les échanges de marché, plus que toutes autres activités, sont déterminés par la poursuite délibérée et calculée d'intérêts personnels. Ils sont libres des diverses emprises de la tradition et des caprices des influences sentimentales. ${ }^{15}$

Avant l'arrivée des Européens, les Kanaks de la Nouvelle-Calédonie étaient engagés dans une agriculture de subsistance, cultivant les ignames et irrigant les taros dans les vallées et sur le flanc des coteaux. De plus, ils pratiquaient la pêche, la chasse et la cueillette dans les forêts et sur le bord de la mer. Les habitants des vallées participaient dans un réseau d'échanges de produits avec les groupes côtiers. Le pays tout entier était traversé de systèmes complexes de mariages et d'échanges traditionnels.

Aux temps modernes, les Kanaks continuent d'effectuer des échanges traditionnels. Cependant, les articles maintenant échangés sont de l'argent liquide, des étoffes et du tabac. Néanmoins, les valeurs et les institutions kanakes, bien que modifiées par le colonialisme, continuent à servir de point de référence pour tous les aspects de la vie sociale. Elles demeurent la base de l'identité kanake et sert de point de mire dans la bataille pour l'indépendance. Nous le constatons ci-dessous dans la citation de Jean-Marie Tjibaou - au Conseil du gouvernement de la Nouvelle-Calédonie de 1982 au 1986, et à la tête du mouvement indépendantiste jusqu'à sa mort en 1989:

\begin{abstract}
Les expressions changent selon la période et l'environnement. Cependant, le sens de ces réponses, la signification de notre concept de l'homme et de son rapport avec l'espace, le cosmos et le monde demeure le même. Pour réaliser la tradition, nous avions l'habitude d'utiliser la monnaie kanake. Aujourd'hui, nous ajoutons des étoffes et de l'argent, mais le discours demeure identique. Les références philosophiques et nous ajouterions que les références socio-mythiques demeurent les mêmes. Ceci est primordial, ça n'a pas changé, et je crois que ça durera toujours. ${ }^{16}$
\end{abstract}

Pareillement, le système technico-culturel des Kanaks a donc subi une évolution même si l'introduction de nouveaux matériaux et de nouveaux outils n'a que très peu affecté le système technique. Pendant qu'ils appréciaient les bénéfices des nouveaux outils, ils contrôlaient l'objet étranger en le transformant en une forme et un usage conformes à leurs traditions. Ce maintien des traditions était non seulement pratiqué avec la technologie mais aussi avec l'usage de cette technologie. Donc, pendant les premières années de contact, aucun auteur ne remarque une expansion de la chasse ou un

14. Economy and Society, Guenther Roth and Claus Wittich éds, Vol. 2. Berkeley: University of California Press. 1978, p. 635-p 636, traduction.

15. Roger Brubaker, The Limits of Rationality, London: George, Allen \& Unwin, 1984, p.10.

16. Jean-Marie Tjibaou, 1988, cité dans Banian, No 5, 1990, p.10. 
accroissement de l'agriculture dûs à l'adoption de la nouvelle technologie. ${ }^{17}$ Pareillement il est possible d'exploiter des mines de nickel pour l'amélioration de la condition du peuple kanak, de la même façon qu'une personne utiliserait son propre jardin pour y faire pousser des tubercules pour le bien du clan.

Les dirigeants kanaks ont été très clairs en affirmant qu'ils sont opposés à l'idée du "profit individuel." C'est l'une des raisons pour lesquelles le mouvement d'indépendance kanake, le Front de Libération Nationale Kanak et Socialiste (FLNKS) garde l'idée d'une indépendance socialiste même si ce n'est pas le socialisme que l'Ouest est habitué de rencontrer. Dans le passage suivant, Jean-Marie Tjibaou explique le sens du socialisme pour la Kanaky - le nom que le FLNKS a choisi pour une Nouvelle-Calédonie indépendante.

Le S dans FLNKS signifie que la souveraineté de notre terre se traduit par une organisation économique, par un refus de l'exploitation de notre patrimoine exclusivement pour le profit d'un petit nombre de personnes. Pour nous, ce S est aussi intimement lié à Kanaky. Il n'y a aucune référence que ce soit aux doctrines développées par d'autres. Nous écrirons nous-mêmes notre socialisme. ${ }^{18,19}$

L'argent provenant des mines a besoin d'être utilisé pour le bénéfice de tous. Tjibaou poursuit:

Pour moi, extraire des minéraux d'une montagne et l'exporter, c'est une politique "d'accumulation." Le territoire n'a jamais vraiment eu une politique concernant l'industrie minière. Ce patrimoine se doit d'enrichir la collectivité, soit à l'intérieur du capitalisme, soit dans une dimension socialiste. C'est maintenant le temps pour la Nouvelle-Calédonie de créer une stratégie de développement dans laquelle le patrimoine minier est clairement défini comme un véritable outil de développement pour le territoire. $^{20}$

Faisons ici un historique de la participation récente des Kanaks au développement minier du territoire. Pendant les années 80 , le territoire connut une période très violente blocages de routes, fusillades et destruction des propriétés privées - qui, à la veille des élections présidentielles en France en 1987, culmina en une dramatique prise d'otages. Dans un effort pour éviter plus de violence, le premier ministre français réunit les membres du FLNKS et ceux du parti conservateur des colons (le RPCR) pour décider de l'avenir du territoire. Les résultats de ces négociations sont connus sous le nom des accords de Matignon.

Les accords annonçaient une période de paix de dix ans durant laquelle le gouvernement français s'efforcera de redresser les inégalités socio-économiques, particulièrement en promouvant le développement et les programmes d'éducation dans les

17. Isabelle LeBlic, Les Kanak Face au Développement, Nouméa: ACDEK, 1993, p.131.

18. Jean-Marie Tjibaou, 31 janvier 1986.

19. Ceci est intéressant en ce que le socialisme comme il existe en Europe est une autre variante du rationalisme formel, en ce sens que des buts quelques peu différents sont poursuivis avec les mêmes moyens technologiques, une discipline d'usine, et des organisations bureaucratiques hiérarchisées (Raymond Murphy, "Rationalism and Ecological Rationality," mimeo, Université d'Ottawa, 1993, p.13).

20. Jean-Marie Tjibaou, "Mon idée de développement," 30 Jours, octobre 1993, p. 14. 
communautés kanakes. En 1998, à la fin de ces dix années les citoyens de la NouvelleCalédonie (dont les Kanaks constitueront 52\%) seront appelés à choisir entre l'indépendance et le maintien du statut de territoire français.

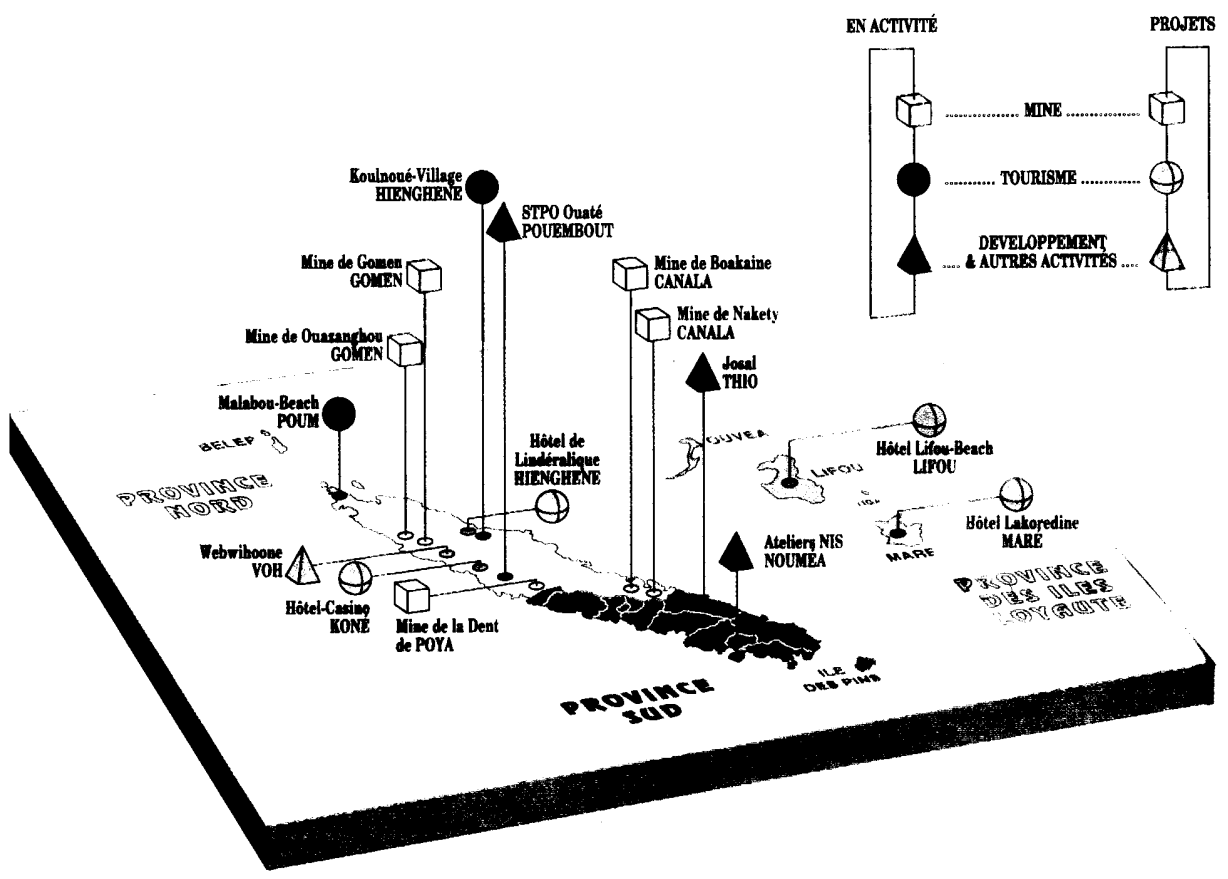

Figure 2. Carte des Implantations du Groupe en Nouvelle-Calédonie. Source: Groupe Sofinor, Rapport d'activité. Nouméa Nouvelle-Calédonie, 1991.

Le territoire a été divisé en trois régions - nord, sud et îles - et les Kanaks contrôlent le nord rural et la région des îles où ils représentent la majorité de la population. Les structures bureaucratiques ont été décentralisées pour mieux servir les régions et la priorité fut donnée à la formation de cadres kanaks. De façon à créer un équilibre entre les régions kanakes et la région du sud, urbaine et à prédominance blanche, le nord et les îles ont bénéficié de $75 \%$ du budget des investissements publics du territoire. Avec cet argent les provinces Nord et Iles ont mis en place des projets de développement. Pendant que la France mise sur ces dix années pour démontrer aux Kanaks la nécessité de rester dans la république française, les Kanaks sont déterminés à utiliser cette période pour acquérir les compétences, expertises et infrastructures nécessaires à l'indépendance.

En septembre 1990, partie intégrante des accords de Matignon, la province Nord, dominée par les Kanaks, créa une compagnie du nom de SOFINOR (La Société de Financement et d'Investissement de la Province Nord). La compagnie a été conçue comme "outil de développement" qui investirait dans les mines et la métallurgie, l'industrie agricole, les pêches et le tourisme. Le premier achat d'importance de SOFINOR fut des parts d'une compagnie minière et de mines de nickel (SMSP - La Société Minière du Sud Pacifique) appartenant à un éminent homme d'affaires local. Par la suite, SOFINOR 
acheta une compagnie d'exportation de marchandises - transportant principalement du nickel par voie maritime - et deux compagnies touristiques.

En 1991, SOFINOR acheta un droit d'accès à deux autres sites miniers, augmenta ses parts dans SMSP à 95\% pendant que la province des îles, aussi dominée par les Kanaks, s'accapara les 5\% restants. SOFINOR construisit dans le nord un centre de villégiature 5 étoiles (possession de SOFINOR à 81\%) et participa (à 77\%) à la construction d'un Club Med, encore une fois dans la Province Nord. Le but principal de ces développements touristiques est d'accroître l'activité économique de la population locale, notamment via le recrutement de personnel kanak et par l'achat de nourriture et autres biens provenant de fournisseurs locaux. Dans la construction de l'Hôtel Malibou et celle du Club Med, les aînés tribaux ont été consultés dès l'étape de la planification. Les aînés étaient déterminés à ne pas compromettre la tradition. Par exemple, on ne retrouve aucune discothèque sur les sites. Cela décourage les jeunes Kanaks de se tenir près des hôtels et d'y boire. Cette consultation a été organisée par les dirigeants de SOFINOR qui sont pour la plupart des indépendantistes kanaks.

D'autres investissements de SOFINOR comprennent $46 \%$ des parts d'une ferme d'élevage de crevettes visant l'exportation et la possession d'une compagnie de machinerie lourde (utilisée pour la construction de routes et dans les mines). ${ }^{21}$ L'argent de ces grandes compagnies est ensuite utilisé pour financer de plus petits projets de développement. Le but, alors, est de créer un environnement fertile pour l'indépendance par l'expérience acquise dans l'administration de ces projets.

SOFINOR entreprend aussi des actions pour s'assurer que la pollution soit adéquatement contrôlée dans ses mines. L'environnement est récemment devenu une préoccupation $\mathrm{du}$ mouvement indépendantiste kanak. Pour ce faire le front indépendantiste présente ses candidats aux élections provinciales qui, une fois élus, nomment les directeurs des compagnies, comme SOFINOR, sous le contrôle de la province. Le FLNKS espère ainsi pouvoir diriger le développement du territoire. Lors d'une récente entrevue, le chef actuel du FLNKS, détenteur d'un diplôme d'économie d'une Université française, Paul Neaoutyine, a révélé:

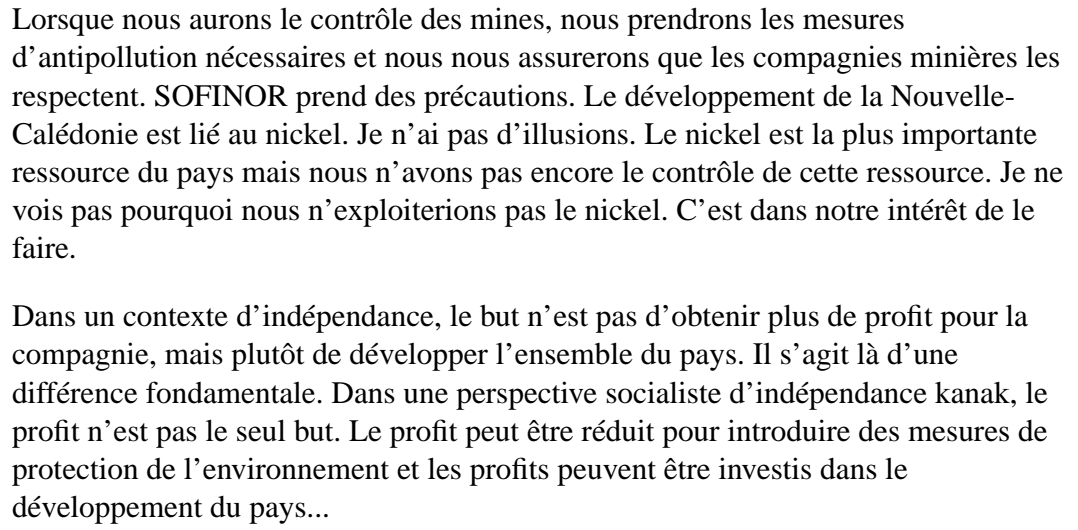

Dans un contexte d'indépendance, le but n'est pas d'obtenir plus de profit pour la compagnie, mais plutôt de développer l'ensemble du pays. Il s'agit là d'une différence fondamentale. Dans une perspective socialiste d'indépendance kanak, le profit n'est pas le seul but. Le profit peut être réduit pour introduire des mesures de protection de l'environnement et les profits peuvent être investis dans le développement du pays...

Pour nous, la préoccupation environnementale est importante parce que, par le biais du développement économique, nous pourrons utiliser les profits pour améliorer les

21. Groupe SOFINOR, Rapport d'activité 1991. Nouméa: SOFINOR. 
conditions de vie ainsi que la qualité de l'environnement... En ce sens, parce que nous sommes pour le socialisme, nous ferons le nécessaire pour que les résultats miniers soient utilisés au développement de toute la société, comme pour les programmes socioculturels ainsi que pour contrôler la pollution et protéger l'environnement. ${ }^{22}$

De plus, la direction kanake de SOFINOR respecte les traditions du peuple kanak. Par exemple, lorsque SOFINOR voulait poursuivre le développement du nickel dans la région de Waco, le clan Ahrou de la ville de Koumac a été capable d'empêcher l'exploitation de certains sites sacrés. A Boakaine, une zone minière de la région de Canala, une filiale de SOFINOR La Société Minière du Sud Pacifique (SMSP), a dû entreprendre de longues discussions avec les autorités traditionnelles de la région parce que le propriétaire précédent de la mine (la "Société Le Nickel", contrôlée par la Banque Rothschild qui a été nationalisée par le gouvernement français) a pollué la région par une exploitation irréfléchie du site. Selon le président de SOFINOR, Raphaël Pidjot (fils d'un des premiers indépendantistes kanaks), les références culturelles furent placées au premier plan. Les autorités traditionnelles ne voulaient pas la même sorte d'exploitation, et on en vint à une entente selon laquelle la population locale serait associée avec le SMSP dans ses opérations. Toutes les relations entre le SMSP et la population locale s'effectuaient avec l'aide des autorités traditionnelles comme médiateur. ${ }^{23}$ Vingt pour-cent des profits vont aux gens de Canala. Selon le président de SOFINOR, "Si, pendant les cinq dernières années, d'autres personnes avaient fait ce que nous avons fait, l'apparence du territoire serait totalement différente. Si d'autres mines avaient investi dans le territoire au lieu de retirer les profits du pays et de les investir ailleurs." 24

Nous avons vu plus haut que la rationalité capitaliste formelle, où les activités économiques sont déterminées par le calcul des intérêts personnels, s'oppose aux activités économiques kanakes qui déterminent, quant à elles, une rationalité substantive qui s'appuie sur la valeur des fins, ici utiles à la communauté kanake.

\section{b. La loi rationnelle et l'administration bureaucratique}

Passons au second critère de rationalisme occidental selon Weber. La loi et l'administration bureaucratique sont rationnelles à cause de leur forme objectivée, institutionnalisée et supra-individuelle.

Si le capitalisme constitue l'une des deux principales références empiriques de la conception Weberienne du "rationalisme spécifique et particulier de la culture occidentale", la loi rationnelle et l'administration bureaucratique ensemble contiennent l'autre. ${ }^{25}$ Nous n'entrerons pas ici dans les détails à propos de l'imposition d'une administration coloniale en Nouvelle-Calédonie. Plutôt, nous nous concentrerons sur la loi kanake et nous ferons référence à la loi française concernant les mines où les règlements formels définissent le "domaine juridictionnel" de chaque agence, en spécifiant la distribution de l'autorité. Donc, sous la loi coloniale, le Ministère de l'industrie, et non les

22. Paul Neaoutyine, communication personnelle, 12 juin 1993.

23. Raphaël Pidjot, communication personnelle, 15 juin 1993.

24. Raphaël Pidjot, communication personnelle, 15 juin 1993.

25. Roger Brubaker, The Limits of Rationality, London: George, Allen \& Unwin, 1984, p.16, traduction. 
autorités traditionnelles de clan, a autorité pour déterminer qui peut développer les flancs des montagnes.

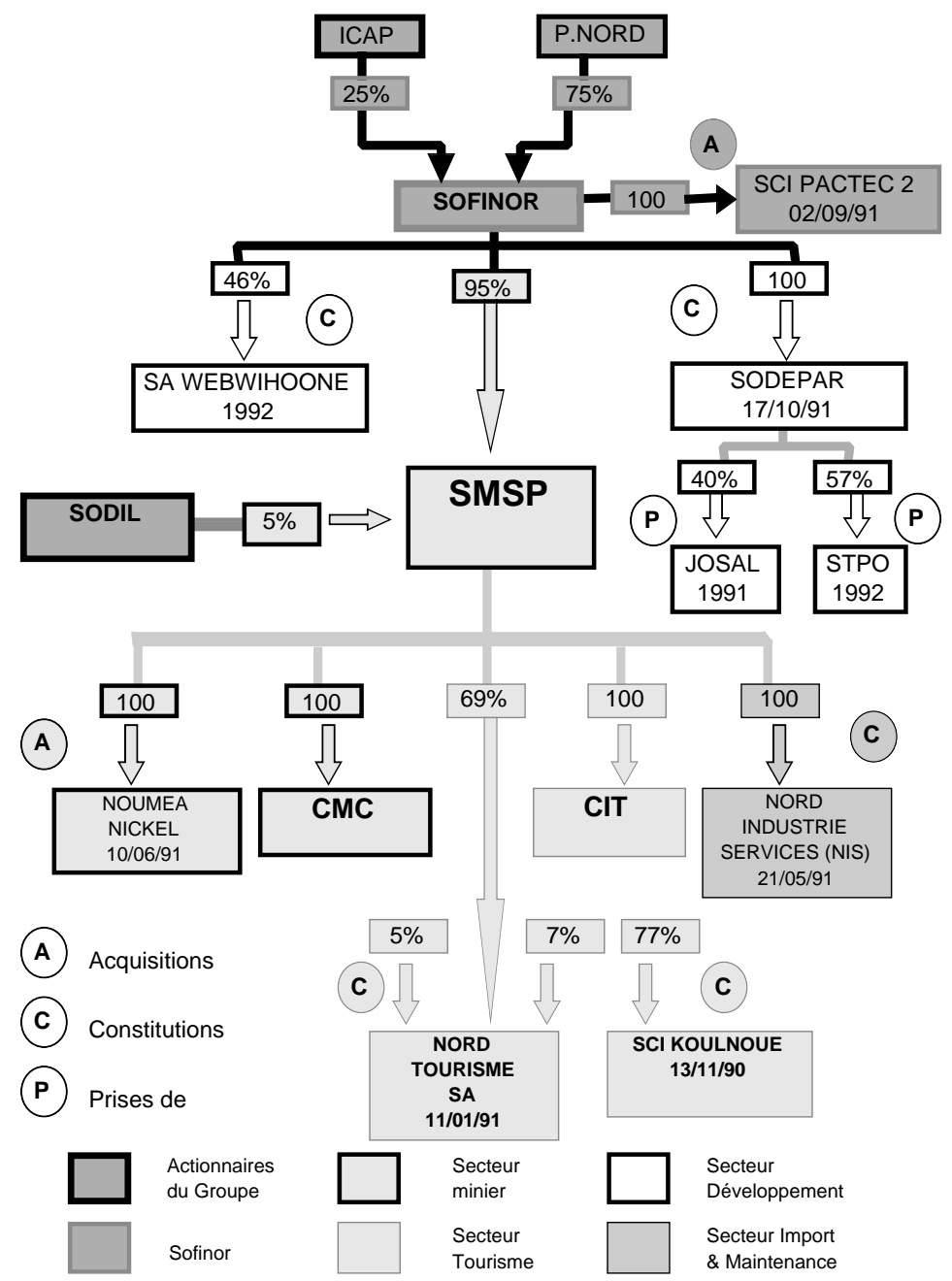

Figure 3. L'Organigramme Actuel. D'après l'organigramme de SOFINOR, 1991. Source: Groupe Sofinor, Rapport d'Activité. Nouméa Nouvelle-Calédonie, 1991.

La terre représente un élément clé dans l'identité kanake et selon Saussol, "toute l'histoire coloniale en Nouvelle-Calédonie en est une où la lutte pour la terre est au centre des débats." ${ }^{26} \mathrm{La}$ terre n'est pas une propriété avec ses frontières. C'est l'approvisionnement en nourriture pour le clan, la commémoration de la tradition et la fondation de l'identité kanake qui est une sorte d'auto-concept foncier. Chaque nom de clan représente un toponyme - le nom d'un site où l'ancêtre du clan s'est manifesté ainsi

26. Alain Saussol, Les Temps Modernes, 1985, p. 1612. 
que tous les sites successifs occupés dans l'histoire du clan. La terre est inaliénable parce qu'elle n'appartient pas à l'homme, mais l'homme appartient à la terre. Selon Jean Marie Tjibaou "La terre n'est pas un capital qui est objectif et qui a une distance par rapport au groupe humain, à l'homme." 27 Ceci rappelle les mots de Roch Pidjot, père indépendantiste de l'actuel président de SOFINOR, dont nous avons parlé plus tôt: "La terre est première, elle est nous. Sans les retours de notre terre, nous demeurons un peuple sans racine et sans notre identité." 28 Cette vision contredit la rationalité des colons européens qui voient la terre comme un objet économique et comme preuve tangible de leur conquête et de leur occupation coloniale. ${ }^{29}$

La nature des liens généalogiques des clans et la distribution de leurs sites d'émergences, mémorisés dans les mythes, établit des relations sociales entre personnes et groupes. De façon similaire, la loi traditionnelle kanake était gouvernée par les esprits des ancêtres qui, avec leurs pouvoirs de sanctions et d'autorité coercitive, limitaient les transgressions. Ils protègent les droits sur les récoltes de jardin, les zones de pêche et les lieux résidentiels en s'assurant que les gens respectent les règles et tabous.

Les ancêtres sont les gardiens des montagnes, des tertres familiaux et des cimetières de leur groupe paternel. Ils défendent les droits de propriété de leurs descendants en punissant les contrevenants qui ne respectent pas la terre ou les droits des descendants à la terre. Les maladies physiques ou même la mort peuvent terrasser les transgresseurs. Or, en 1987, lorsque les policiers français poursuivaient les nationalistes kanaks à travers la brousse, ils étaient retrouvés plusieurs jours plus tard, perdus, nus et le visage hagard. Il était dit que c'était le travail des esprits de la forêt. ${ }^{30}$ Les ancêtres sont omniprésents et leur présence est ressentie dans la pluie lorsqu'elle tombe, dans le passage d'un insecte, le regard d'un chien, etc. Ils peuvent apparaître sous des formes d'animaux, de plantes, de roches, du vent, du tonnerre et même d'une vague. Les ancêtres du clan sont créés à partir de forces naturelles. Par exemple, un vers de terre frappé d'une lumière signifie qu'un ancêtre en émerge. ${ }^{31}$ Un requin épouse une moule, un rocher s'accouple avec une huître, le tonnerre avec une anguille, le vent avec une buse. Chaque clan voit l'expression de ses ancêtres dans la nature, ce qui leur permettent de se distinguer des autres. Les ancêtres résident de façon permanente dans les éléments, plantes, animaux et dans certains sites spécifiques. ${ }^{32} \mathrm{Il}$ est donc essentiel que l'industrie minière prenne en considération l'utilisation de l'environnement naturel autant dans un sens sacré qu'écologique, puisque

27. Jean-Marie Tjibaou "Etre Mélanésien Aujourd'hui", Esprit, 1981, p.89.

28. Roch Pidjot, 18 décembre 1984, cité dans LeBlic, Les Kanak Face au Développement, Nouméa: ACDEK, 1993, p.26.

29. Alain Saussol, Les Temps Modernes, 1985, p. 1612, Isabelle LeBlic, Les Kanak Face au Développement, Nouméa: ACDEK, 1993, p. 26.

30. Alban Bensa, "Des ancêtres et des hommes." Dans: De Jade et de Nacre, Paris: Réunion des musées nationaux, 1990: 131.

31. La métaphore en est une ici de reproduction, la lumière du mâle allant vers la femelle pour créer l'ancêtre du clan Dulo Jaai. Les descendants de ce clan se référeront à l'un ou à l'autre des esprits des ancêtres, selon leur genre. Voir Alban Bensa, "Des ancêtres et des hommes". Dans De Jade et de Nacre, Paris: Réunion des musées nationaux, 1990:137. 
le caractère sacré d'un site ne peut pas en être dissocié. C'est très différent des droits de propriété sous les lois françaises.

Les lois concernant les mines de Nouvelle-Calédonie sont une extension des lois françaises - les droits du sous-sol appartiennent à l'état. Les droits d'exploration sont différents devant la loi des droits d'exploitation et chacun demande un permis différent. Depuis 1969 le pouvoir de distribution de ces autorisations pour le nickel, le chrome et le cobalt, repose entre les mains du Ministre français de l'industrie et non entre les mains des autorités locales. Pour la France, le nickel est un minerai stratégique puisque c'est un élément essentiel dans la production d'armes, d'électronique, d'avions ainsi que dans la production nucléaire, et cela sans compter les chars d'assaut et les canettes de bière. Par sa présence continue en Nouvelle-Calédonie, la France réduit sa dépendance sur les sources étrangères et a ainsi contrôlé une large proportion des réserves mondiales de nickel. En avril 1990, Louis Le Pensec, Ministre du département d'Outre-mer et des Territoires, a souligné que le nickel de la Nouvelle-Calédonie était "en quantité suffisante pour constituer un enjeu économique de taille pour la métropole française."33

Selon la loi civile française, la terre est considérée comme une chose nommée propriété. Donc la possession de la terre est un droit réel sous la loi, alors que:

Selon la tradition kanake, les relations entre les hommes kanaks et la terre et l'espace, par le clan, ses alliances et son totem, ne sont plus quelque chose que nous avons à prouver. Chaque personne est originaire de quelque part et va quelque part en suivant les traces de son clan. C'est pourquoi les Kanaks en général ont tendance à dire notre terre, notre patrimoine ou "c'est la terre de nos ancêtres que nous ravageons - nos anciens, nos grands-parents." Cette référence historique à nos anciens et nos grandsparents est une expression du groupe autant que du clan puisque cela démontre de notre communauté et de notre nationalité avant l'arrivée des blancs. Il est important d'insister sur cette référence historique. Notre héritage naturel, et conséquemment les mines, ne peut pas être national avant d'être clanique. "C'est la terre de nos ancêtres, de nos grands-parents.” Cela signifie aussi que c'est notre terre, notre terre à la lumière d'une expérience coloniale commune. Quand le FLNKS déclare que le soussol constitue notre héritage national et reconnaît les droits des clans comme une voie concrète vers l'obtention du développement économique, nous ne trahissons pas le peuple kanak. ${ }^{34}$

32. Les ancêtres participent également à la formation d'un chef de façon similaire au processus d'élaboration d'un jardin ou encore comme on élève un enfant. Le "chefenfant" est un étranger que le groupe a accueilli sur leurs terres qui sont protégées par les génies et les ancêtres du clan local, et ces forces supernaturelles contribuent à l'énergie du chef. La convergence de ses efforts est réalisée sur le plan matériel par la construction d'une maison splendide, d'une allée centrale menant à cette demeure, d'objets prestigieux (poutres de faîte/ de sommets/ de flèches, de masques de haches de cérémonies) et par un conseil de bande. Les rituels obligent le chef, un nouveau "fils," à ses sujets et à leurs ancêtres (Alban Bensa "Des ancêtres et des hommes." Dans: De Jade et de Nacre, Paris: Réunion des musées nationaux, 1990:140-41).

33. Cité dans S. Henningham, "France and the South Pacific in the 1980s: An Australian Perspective," Journal de la Société des Océanistes, Vol 92-93, 1991, p.22, traduction.

34. PALIKA, dans Kanak, No 149, sept. 1992. 


\section{c. Orientation interne rationnelle}

Selon Raymond Murphy, un autre spécialiste de Weber, l'élément central au dynamisme du rationalisme formel est "l'intellectualisation": le développement de la compréhension de la nature et comment la manipuler. "L'intellectualisation résulte de toute façon en la perte de l'ancien sens spirituel dominant de la vie... La rationalisation, et l'intellectualisation en particulier, ont promu la croyance que la réalité peut être reconstruite par la société humaine, et donc la perte de l'attitude qui veut que l'homme doive s'adapter à la nature."35 Cette vue est vraiment loin de la perspective kanake des relations humaines vis-à-vis de l'environnement. J'aimerais présenter une série de citations de Jean- Marie Tjibaou qui expliquent cette perspective de façon très éloquente :

Et cette réalité-là, elle est exprimée d'une façon qui doit tenir compte de l'environnement, de l'histoire. C'est ainsi que l'on pourrait dire, d'une manière tout à fait banale, que l'homme produit la société, et que la société usine à son tour l'homme. Et, jeté dans le monde des vivants, le petit homme est somme de besoins et d'aspirations, besoin de se nourrir, de s'abriter, d'être en sécurité, de se vêtir, de parler, d'échanger, de se perpétuer par la création, par la procréation; et la société qui l'accueille, offre une somme de réponses liées à l'environnement écologique, géographique, climatique, historique...

Il y a un certain type d'homme qui est fabriqué par l'université, fabriqué par l'école moderne... Comment est conçu l'homme dans le monde mélanésien? Comment est conçu l'univers dans lequel il se trouve? Quels sont les modèles d'homme, les modèles de réussite de l'homme, les rapports de cet homme avec l'univers des vivants, avec les êtres vivants, avec le terroir? Je pense que les gens qui sont encore de la terre, qui sont des ruraux, peuvent communier facilement avec ce que je veux dire. Quels sont les rapports avec le soleil, la lune, le tonnerre, les saisons? Et les rapports avec ceux qui vivent? Je dirais: "Au-delà du miroir". Tout ceci pour vous donner un peu un profil de l'homme traditionnel: c'est celui qui sort de la terre, de l'humus, de la tribu, qui circule en ville mais qui n'est pas un produit de l'université, qui est un produit de la terre, un produit de la tribu.

Et la réponse à la question “d'où venons- nous?", sur la terre de Calédonie, les gens l'ont traduite dans les généalogies. C'est "les gens de tel clan,... c'est le clan...ils sortent de la paille, tel genre de paille, poussée sur telle montagne, et de cette paille est issu tel clan, et dans ce clan... Rappelez-vous, ceux qui connaissent la Bible, la généalogie de Jésus, 'fils de David, fils d'Abraham, fils d'Adam'... Nous retrouvons le même schéma. Mais nous trouvons, au terme, un arbre, ou un animal, ou une pierre, ou le tonnerre. C'est la relation avec la terre, avec l'environnement, avec le pays, avec le terroir. Nous ne sommes pas des hommes d'ailleurs. Nous sommes des hommes sortis de cette terre."

Le principe de vie, nous disons que c'est la mère qui donne la vie. Le père donne le personnage, le statut social, la terre... Alors je reste toujours duel Je ne suis jamais individu. Je ne peux pas être individu. Le corps n'est pas un principe d'individuation. Le corps est toujours la relation.

35. Raymond Murphy, "Rationalism and Ecological rationality," mimeo, Université d'Ottawa, p. 7,14, traduction. 
Dernièrement j'ai participé à une session de commission du Conseil oecuménique des Eglises sur les atteintes aux droits de l'homme. Droit de liberté, d'expression... Les violations des droits, c'est aussi sur les terres, et je disais que c'est inscrit dans la genèse: la philosophie judéo-chrétienne parle de Dieu qui créa le monde, vit que tout était bon, etc., et puis il montra la terre à l'homme, il dit: "Croissez et multipliezvous" et puis: "Dominez la terre.” Dominer la Terre? Il est le maître. Dans notre système, l'homme n'est pas le maître. Il est un élément du monde. Il est parmi les plantes. Il y a une plante qui est son totem. Parmi les animaux, il y a un animal qui est son totem, qui est le totem d'un autre clan. Et c'est toute une autre philosophie qui est branchée là-dessus. Nous faisons partie du monde, du monde des vivants, du monde de la nature, du monde des arbres, des plantes, des pierres, et il faut le respecter. ${ }^{36}$

Ces passages qui peuvent paraître idéalistes aux yeux d'un Occidental, représente la façon de vivre et de ressentir l'environnement dans la culture traditionnelle kanake. Evidemment il y a eu des adaptations nécessaires à l'aboutissement de l'indépendance et de la reconnaissance de la culture kanake, changements perceptibles dans la participation politique et économique du peuple kanak tant au niveau de la gestion des provinces Nord et des Iles que dans la gestion de SOFINOR qu'ils essaient de rendre plus respectueuse de l'environnement et de la culture kanake.

\section{Conclusions}

En Nouvelle-Calédonie, le savoir des aborigènes était traditionnellement ancré dans un jugement substantif de l'environnement naturel, caractérisé par une conviction de la valeur intrinsèque d'un objet ou d'une action. Lorsque la France annexa la NouvelleCalédonie, elle imposa un système de relations capitalistes de production avec un colonialisme basé sur l'immigration, l'exploitation des ressources minières et les fermes d'élevage. Cette façon d'opérer est responsable de l'introduction d'une rationalité instrumentale ou formelle caractérisée par un raisonnement conscient qu'une action ou un objet est un moyen de parvenir à ses fins. L'environnement naturel se transforme alors en un moyen et un instrument servant consciemment à poursuivre des projets pour les profits qui furent anticipés par les colonisateurs. En même temps, la majorité des Kanaks continuent d'apprécier la valeur intrinsèque de la nature puisqu'ils ont été exclus du secteur capitaliste de l'économie de la Nouvelle-Calédonie en vivant à l'intérieur de réserves aborigènes et continuant leurs activités de subsistance.

Depuis les vingt dernières années, on observe l'émergence d'une élite kanak qui adopte une valeur de la nature comme moyen de parvenir à leurs buts - s'affranchir de la France. L'utilisation de l'environnement est maintenant perçue comme une façon de concrétiser un objectif spécifique, par exemple s'approprier les titres de propriété des mines de nickel pour obtenir leur indépendance économique. Ici j'ai essayé demontrer que le développement de l'environnement de la Nouvelle-Calédonie, pour les Kanaks nationalistes, est mené principalement à travers une rationalité substantive, c'est-à-dire dans le but spécifique de l'indépendance des Kanaks et du développement du peuple kanak.

En plus, pour Weber, la rationalisation ne représente pas un simple procédé mais plutôt une multitude de précédés distincts, quoiqu'inter-reliés, émergeant de différentes sources historiques et agissant à différents niveaux. En Nouvelle-Calédonie, la

36. Jean-Marie Tjibaou, "Etre Mélanésien Aujourd'hui,” Esprit, 1981, pp. 81-93. 
rationalisation formelle fut concentrée dans les zones urbaines à prédominance européenne. Les Kanaks sont demeurés isolés des forces du marché, accompagnant ainsi les procédés formels rationnels en continuant la pratique de l'agriculture de subsistance dans les réserves. Il reste à voir pour combien de temps ils seront capables de continuer de cette façon. Les attraits des villes et du marché capitaliste ont actuellement un impact important sur la jeunesse kanake. Bienque pour l'instant, la rationalité substantive domine encore les régions kanakes.

Comme le procédé de rationalisation s'intensifie en raison d'une participation accrue à l'économie de marché et à l'éducation occidentale, la question demeure à savoir quelles valeurs et prémisses seront utilisées pour superviser et diriger le cours de l'avenir de la Nouvelle-Calédonie. Ceci ne pouvant être déterminé que par le peuple kanak lui-même. Le conflit portant sur le contrôle des ressources, particulièrement à propos de la terre dans le territoire, constitue en partie un conflit culturel sur les modèles qui seront utilisés pour guider le pays vers le 21e siècle. Le débat continue non seulement entre les Européens et les Kanaks mais aussi entre les Kanaks eux-mêmes afin que la tradition kanake puisse continuer de modeler le futur: "Nous voulons autre chose: pas le retour au passé pour y rester, mais pour retirer du passé la force et les références qui supporteront nos paroles dans le présent et le futur." ${ }^{37}$

\section{Abstract:}

In New Caledonia, indigenous knowledge was traditionally anchored in a substantive appreciation of the natural environment. Substantive, in this case, refers to the Weberian concept of substantive or value rationality characterized by a belief in the intrinsic value of an object or action. When New Caledonia was annexed by France, the imposition of capitalist relations of production through settler colonialism, mineral exploitation and ranching introduced instrumental or formal rationality characterized by conscious reasoning that an action or object is a means to a particular ends. The natural environment became a means and an instrument to carry out conscious projects for the anticipated benefit of the colonizers. At the same time, the majority of the Kanaks continued to appreciate the intrinsic value of nature because they were excluded from the capitalist sector of the New Caledonian economy, living in native reserves and continuing in subsistence activities which made light use of the environment. However, within the past twenty years an emerging Kanak elite has adopted a value of nature as a means to achieve a particular end - independence from France. Rational use of the environment is now perceived as a way to achieve a specific goal, for example, owning nickel mines to achieve economic independence. Maintaining the tension between Weberian concepts of substantive and formal rationalism, this paper traces the emergence of rationalist thought about the environment among Kanak independence leaders.

Keywords: New Caledonia, Kanak, Weber, environment, capitalism, mines, indigenous knowledge, rationalism.

\section{Resumé:}

En Nouvelle-Calédonie, le savoir des aborigènes était traditionnellement ancré dans un jugement substantif de l'environnement naturel. Dans ce cas-ci, nous employons le terme

37. Jean-Marie Tjibaou, "Etre Mélanésien Aujourd'hui", Esprit, 1981, p.82. 
substantif en faisant référence au concept substantif ou valeur de rationalité de Weber, caractérisé par une conviction de la valeur intrinsèque d'un objet ou d'une action. Lorsque la France annexa la Nouvelle-Calédonie, elle imposa un système de relations capitalistes de production avec un colonialisme basé sur l'immigration, l'exploitation des ressources minières et les fermes d'élevage. Cette façon d'opérer est responsable de l'introduction d'une rationalité instrumentale ou formelle caractérisée par un raisonnement conscient qu'une action ou un objet est un moyen de parvenir à ses fins. L'environnement naturel se transforme alors en un moyen et un instrument permettant de poursuivre consciemment des projets pour les profits qu'anticipaient les colonisateurs. En même temps, la majorité des Kanaks continuerent d'apprécier la valeur intrinsèque de la nature puisqu'ils ont été exclus du secteur capitaliste de l'économie de la Nouvelle-Calédonie en vivant à l'intérieur de réserves aborigènes et en continuant leurs activités de subsistance. Quoi qu'il en soit, depuis les vingt dernières années, on observe l'émergence d'une élite kanake qui adopte une valeur de la nature comme moyen de parvenir à leur but - obtenir leur indépendance de la France. L'utilisation rationnelle de l'environnement est maintenant perçue comme une façon de concrétiser un objectif spécifique; l'appropriation de titres de propriété des mines de nickel pour obtenir l'indépendance économique. Tout en entretenant les tensions entre les concepts de rationalisme formel et substantif de Weber, cet écrit retrace l'émergence de la pensée rationnelle et formelle à propos de l'environnement chez les dirigeants kanaks prônant l'indépendance.

Mots Clef:Nouvelle-Calédonie, Kanak, Weber, environnement, capitalisme, mines, savoir aborigène, rationalité.

\section{Resumen}

Nueva Caledonia el saber indígena estaba tradicionalmente basada en una apreciación substantiva del ambiente natural. Substantivo, en esta caso, se refiere al concepto Weberiano de substantivo o valor racional caracterizado por una creencia en el valor intrínseco de un objecto u acción. Cuando Nueva Caledonia fué anexada a Francia, la imposión de relaciones capitalistas de producción a través del colonialism pacificador, la explotación mineral y la ganadería introdujeron la racionalidad instrumental o formal caracterizada por el raciocinio consciente de que una acción o un objecto es un medio para llegar a fines particulares. El ambiente natural se convirtió en el medio e instrumento para llevar a cabo proyectos conscientes para el beneficio anticipado de los colonizadores. Al mismo tiempo, y al ser excluidos del sector capitalista de la economía de Nueva Caledonia, la mayoría de los Kanks continuaron apreciando el valor intrínseco de la naturaleza. Así continuaron viviendo en las reservas nativas y continuaron sus actividades de subsistencia las cuales hacían menor uso del medio ambiente. Sin embargo, en los últimos veinte años surge una élite Kanke que ha adoptado un valor de la naturaliza como forma para obtener un fin particular--independizarse de Francia. El uso racional del medio ambiente es ahora percibido como una forma para obtener el objecto específico, por ejemplo, ser propiertario de minas de niquel para así poder lograr una independencia económica. Manteniendo la tensión entre conceptos Weberianos de racionalismo substantivo y formal, este artículo rastrea el surgimiento del pesnsamiento racionalista en relación al medio ambiente dentro de los líderes independentistas de Kanak.

Palabras claves: Nueva Caledonia, Kanak, Weber, medio ambiente, capitalismo, minas, saber indígena, racionalismo. 